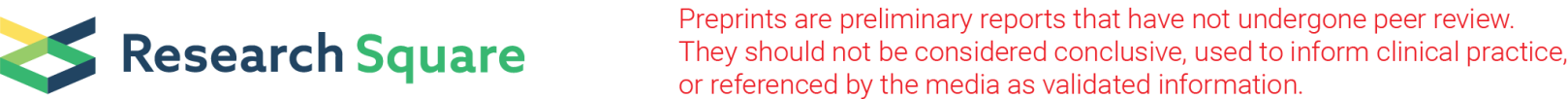

\section{Integrative and Comparative Genomic Analysis and the Immune Microenvironment Features of Lung Cancer Patients with Tuberculosis}

\section{Xiaoling Xu}

Zhejiang Cancer Hospital

\section{Chaohui Bao}

Shanghai Jiaotong University: Shanghai Jiao Tong University

\section{Da Chen}

Zhejiang University School of Medicine

\section{Tianxiang Wang}

Zhejiang University School of Medicine

\section{Changchun Wang}

Zhejiang Cancer Hospital

\section{Weimin Mao}

Zhejiang Cancer Hospital

\section{Xiao Hu}

Shanghai Jiaotong University: Shanghai Jiao Tong University

\section{Yang Yang}

Shanghai Jiaotong University: Shanghai Jiao Tong University

Yun Fan ( $\nabla$ fanyun1218@163.com )

Zhejiang Cancer Hospital

\section{Research}

Keywords: Tuberculosis, lung cancer, tumour microenvironment, PD-L1, C1QB

Posted Date: July 9th, 2021

DOI: https://doi.org/10.21203/rs.3.rs-667647/v1

License: (c) (1) This work is licensed under a Creative Commons Attribution 4.0 International License. Read Full License 


\section{Abstract}

Background: Limited information was known because of the low incidence of co-existence with lung cancer and tuberculosis (TB), it remains special challenging populations for clinical management of cancer immunotherapy. Thus, to investigate the difference on tumour immune microenvironment and genomics between patients with LC alone and LC patients with TB is urgently needed.

Methods:Tumour specimens were collected from 87 patients who had LC, with or without TB, at two medical centres. Immunohistochemistry was used to evaluate PD-L1 expression and CD3+/CD4+/CD8+ T-cell infiltration. Whole-exome sequencing was performed using samples from 19 patients with LC\&TB and 21 patients with LC.

Results:Relative to patients with LC alone, patients with LC\&TB had lower PD-L1 expression and CD4+/CD8+ T-cell infiltration (all $P<0.001$ ). A tumour microenvironment with no PD-L1 expression and CD8- T-cell infiltration was most common in the LC\&TB patients. Genomic alterations analysis revealed an increased mutation frequency among patients with LC and active TB, obsolete/cured TB, or no TB in terms of the TP53 $(23.08 \%$ vs. $66.67 \%$ vs. $76.19 \%, P=0.01)$, while a decreased trend of the number of single-nucleotide variants/insertions/deletions $(P<0.001)$, tumour mutation burden $(P<0.001)$, and number of neoantigens $(P<0.001)$. Patients with LC\&TB had a higher frequency of a specific mutation signature (32.99\% vs. $11.23 \%)$, as well as potential driver mutations involving the complement $\mathrm{C} 1 \mathrm{qB}$ chain $(C 1 Q B)$ mutations.

Conclusion: The present study revealed significant differences in the tumour microenvironment and genomic alterations between patients with LC\&TB and patients with LC alone.

\section{Highlight}

- This study compared lung cancer specimens according to the presence or absence of tuberculosis.

- Lung cancer with tuberculosis had lower PD-L1 expression and less CD3+/CD4+/CD8+ T-cell infiltration.

- Genomic alterations were significantly different according to the presence or absence of tuberculosis.

- Patients with lung cancer and tuberculosis had a unique potential driver gene (C1QB), may influence macrophage and dendritic cell infiltration.

\section{Background}

Lung cancer (LC) remains the major cause of cancer-related death, with an estimated 1.59 million deaths in 2016 , which accounts for approximately $20 \%$ of all cancer-related deaths worldwide (1).

Epidemiological research regarding $L C$ has revealed that the major risk factors are smoking, air pollution, 
occupational factors, and tuberculosis (TB), which will provide the basis for future interventions to improve LC management (2-4).

Mycobacterium tuberculosis (MTB) is the pathogen that causes TB, which is the deadliest infectious disease in the world today $(5,6)$. Infection by MTB affects approximately one-third of the global population, and insufficient treatment or compromised immune defences lead to the development of TB in $5-10 \%$ of infected patients $(5,6)$. Most MTB infections have no symptoms, although TB-induced inflammation typically leads to genetic changes over time, which can drive the development of LC (3). Thus, patients with TB have a significantly increased risk of developing LC and related mortality (3). The increased risk of LC is related to the immunosuppressive state caused by MTB infection (7-9), and coexistence of LC and TB (LC\&TB) has been reported in numerous cases and case-control studies $(3,10$, 11).

Immunotherapy has become a mainstream treatment option for various cancers, including LC and melanoma (12-17), in addition to surgery, radiotherapy, and chemotherapy. Immune checkpoint inhibitors are a type of immunotherapy that activate T-cell responses in the tumour microenvironment and can enhance the immune system's response to the tumour.

Patients with LC\&TB are rare and thus often overlooked in clinical trials, with only limited global data regarding immunotherapy in this patient population (18). Thus, it is unclear whether immune checkpoint inhibitors including anti-PD-1/PD-L1 therapy are contraindicated in patients with LC\&TB. Furthermore, the T-cell response plays a key role in the development and progression of TB (19), and different immune microenvironments of LC patients with and without TB might explain their potentially different treatment responses. Therefore, the present study aimed to evaluate the tumour microenvironment characteristics of LC patients with and without TB, including their PD-L expression and tumour-infiltrating lymphocytes (TILs). Furthermore, we evaluated differences in somatic mutations, copy number variations, tumour mutation burden (TMB), HLA expression, and neoantigen numbers according to the presence or absence of TB.

\section{Materials And Methods}

\subsection{Patient population}

The study was approved by the ethics committees of Zhejiang Cancer Hospital and Affiliated Hangzhou Chest Hospital. This study evaluated data from 87 patients with stage I-IV LC with TB, who were treated between 2013 and 2019 at two Chinese medical centres (Zhejiang Cancer Hospital and Affiliated Hangzhou Chest Hospital). The eligibility criteria were as follows: LC confirmed via pathological or cytological evaluation, complete clinical and follow-up data, availability of $\geq 5$ blank sections from tissue specimens, and no history of malignancy within the previous 5 years. The presence of active TB (ATB) was confirmed based on 3 sputum samples for mycobacterium culture, nucleic acid amplification test and/or lung biopsy (if feasible) (20), while the obsolete TB (OTB) was confirmed based on the patient's 
medical records, the T-cell spot test (TSPOT) or interferon gamma release assay (IGRA), and imaging findings. Cured TB (CTB) was defined as "history of active TB and have been cured when cancer diagnosed". Of note, latent TB was not included in our study. The control group contained stage I-IV LC patients from Zhejiang Cancer Hospital. The study was approved by the ethics committee of Zhejiang Cancer Hospital and Affiliated Hangzhou Chest Hospital.

For genomic analysis, 21 LC\&TB patients, treated with surgery, with more than 15 white paraffin tablets was selected. During 2013-2019, specimens from 192 LC cases had been collected in the hospitals' biobank and we performed propensity score analysis to select 21 paired samples from patients with LC alone. These samples, and normal control tissues, were subjected to whole-exome sequencing (WES) and analysis of copy numbers and somatic mutations. These cancer-specific genomic alterations were then evaluated for associations with the patients' clinical characteristics. The study flowchart is shown in Fig. 1.

\subsection{DNA extraction, exome sequencing, and data processing}

After omitting 2 samples from the LC\&TB group because of poor quality, DNA was extracted from the remaining 40 formalin-fixed paraffin-embedded (FFPE) samples using the QIAamp FFPE DNA Kit (Qiagen, Frankfurt, Germany). The DNA was then fragmented using a Covaris M220 focused ultrasonicator (Covaris, Woburn, USA) and subjected to sequencing library construction. The DNA for WES was captured using the VariantBaits ${ }^{\text {TM }}$ Human All Exon Kit (LC-Bio) according to the manufacturer's recommended protocol. Sequencing was performed using the Illumina NovaSeq ${ }^{\text {TM }} 6000$ system to generate $150-b p$ paired-end reads, and FASTQ software (version 0.20.0) (21) was used to remove low-quality reads and clean the data. The supplementary materials (available online) show the methods for somatic mutation calling, identification of somatic copy number alterations, identification of potential driver genes, extraction of mutation signatures, and prediction of HLA types and neoantigens.

\subsection{Immunohistochemistry}

Immunohistochemistry was used to evaluate the expression of PD-L1 and the presence of tumourinfiltrating $\mathrm{CD}^{+}{ }^{+} \mathrm{T}$-cells, CD $4^{+} \mathrm{T}$-cells, and $\mathrm{CD} 8^{+}$T-cells. We prepared FFPE tissue sections $(4-\mu \mathrm{m})$ on positively charged glass slides, which were stained using primary antibodies targeting CD3 (SP162, 1:150; Abcam, Cambridge, UK), CD4 (B468A1, 1:200; Santa Cruz, TX, USA), CD8 (144B, 1:100; Abcam), PD-L1 (VENTANA PD-L1 [SP263] assay; Roche, AZ, USA), and ALK (VENTANA ALK [D5F3] assay; Roche, AZ, USA). The immunohistochemistry was performed using the BenchMark XT platform and the VENTANA OptiView DAB IHC detection kit. The tumour expression of PD-L1 was evaluated using the tumour proportion score (TPS), which is based on the proportion of tumour cells with positive staining, with a positive result considered present at a PD-L1 TPS of $\geq 1 \%$ (22). The proportions of CD3/CD4/CD8 staining on lymphocytes were evaluated as the proportions of positive cells among all nucleated cells in the stromal compartments, with a positive result considered present at a proportion of $\geq 25 \%$ (22). All 
immunohistochemistry results were judged by two pathologists who were blinded to the patients' clinical characteristics, and disagreements were resolved via discussion. The expressions of PD-L1 and T-cell markers were used to create different subtype groups, and differences between the LC and LC\&TB groups were displayed using stacked bar graphs.

\subsection{Statistical analysis}

All data were expressed as mean \pm standard deviation or number (percentage), unless otherwise indicated. Categorical variables were compared using Fisher's exact test. The Kruskal-Wallis test was used to compare clinicopathological factors, gene alterations, and TMB. Paired groups were compared using the Wilcoxon test, and comparisons of more than two groups were performed using the KruskalWallis chi-squared test. Linear correlations were evaluated using Pearson's correlation coefficient. Most statistical analyses were performed in the R statistical environment (version 3.5.1 or later) and the "survival" package was used to generate and compare the Kaplan-Meier survival curves. The survival event was defined as patients died with lung cancer. GraphPad Prism software (version 8.4; GraphPad Software, San Diego, CA, USA) was used to create the stacked bar graphs. Multivariable survival analyses were performed using the Cox proportional hazards model and propensity score matching analysis was performed using SPSS software (version 24; IBM Corp., Armonk, NY, USA). Differences were considered statistically significant at two-sided $P$-values of $<0.05$.

\section{Results}

\subsection{Clinicopathological characteristics}

The study included 59 patients with LC\&TB (33 patients with ATB and 26 patients with O/CTB) and 28 patients with LC alone. The groups' clinicopathological characteristics are summarized in Table 1 and Supplementary Table S1 (available online). There were no significant inter-group differences in terms of sex, smoking status, histology, pathological T status, lymph node metastasis, distant metastasis, clinical stage, differentiation degree, venous/lymphatic/perineural invasion, adjuvant radiotherapy, adjuvant chemotherapy, or family history of cancer. Most patients had non-small-cell lung cancer (NSCLC, adenocarcinoma or squamous cell carcinoma), although the LC\&TB group included 1 patient with adenosquamous carcinoma, 1 patient with large-cell carcinoma, and 1 patient with small-cell carcinoma. No significant differences in EGFR mutation status were observed between the LC\&TB and LC groups. All tested samples were negative for $A L K$ mutations, although related testing was not performed for $20 \%$ of the patients because of insufficient samples. 
Table 1

The clinicopathological characteristics of entire patient cohort (divided into two groups).

\begin{tabular}{|c|c|c|c|}
\hline Patient Characteristics & LC\&TB group $(N=59)$ & LC group $(\mathrm{N}=28)$ & $P$ value \\
\hline Sex & & & 0.295 \\
\hline Male & $48(81.4 \%)$ & $20(71.4 \%)$ & \\
\hline Female & $11(18.6 \%)$ & $8(28.6 \%)$ & \\
\hline $\begin{array}{l}\text { Median Age } \\
\text { (Range) }\end{array}$ & $62(51-79)$ years & $62(37-75)$ years & \\
\hline Smoking status & & & 0.995 \\
\hline Non-smoker & $19(32.2 \%)$ & $9(32.1 \%)$ & \\
\hline Smoker & $40(67.8 \%)$ & $19(67.9 \%)$ & \\
\hline Histology & & & 0.688 \\
\hline adenocarcinoma & $28(47.5 \%)$ & $12(42.9 \%)$ & \\
\hline Non-adenocarcinoma & $31(52.5 \%)$ & $16(57.1 \%)$ & \\
\hline Pathologic T stage & & & 0.132 \\
\hline $\mathrm{T} 1+\mathrm{T} 2$ & $40(69.0 \%)$ & $22(84.6 \%)$ & \\
\hline $\mathrm{T} 3+\mathrm{T} 4$ & $18(31.0 \%$ & $4(15.4 \%)$ & \\
\hline Lymph node metastasis & & & 0.319 \\
\hline NO & $24(42.1 \%)$ & $15(53.6 \%)$ & \\
\hline N1-3 & $33(57.9 \%)$ & $13(46.4 \%)$ & \\
\hline Distant metastasis & & & 0.931 \\
\hline MO & $44(75.9 \%)$ & $21(75.0 \%)$ & \\
\hline M1 & $14(24.1 \%)$ & $7(25.0 \%)$ & \\
\hline Clinical stage & & & 0.093 \\
\hline I+ II & $22(37.9 \%)$ & $16(57.1 \%)$ & \\
\hline IIII+ IV & $36(62.1 \%)$ & $12(42.9 \%)$ & \\
\hline Differentiation degree & & & 0.865 \\
\hline Poor or undifferentiated & $21(48.8 \%)$ & $6(46.2 \%)$ & \\
\hline Well-intermediate & $22(51.2 \%)$ & $7(53.8 \%)$ & \\
\hline EGFR & & & 0.127 \\
\hline
\end{tabular}




\begin{tabular}{|c|c|c|c|}
\hline Patient Characteristics & LC\&TB group $(\mathrm{N}=59)$ & LC group $(\mathrm{N}=28)$ & $P$ value \\
\hline Positive & $1(5.3 \%)$ & $6(21.4 \%)$ & \\
\hline Negative & $18(94.7 \%)$ & $22(78.6 \%)$ & \\
\hline PD-L1 & & & $<0.001$ \\
\hline Positive & $13(23.2 \%)$ & $18(64.3 \%)$ & \\
\hline Negative & $43(76.8 \%)$ & $10(35.7 \%)$ & \\
\hline CD3 & & & 0.001 \\
\hline Positive & $7(14.6 \%)$ & $11(52.4 \%)$ & \\
\hline Negative & $41(85.4 \%)$ & $10(47.6 \%)$ & \\
\hline CD4 & & & $<0.001$ \\
\hline Positive & $3(6.3 \%)$ & $14(66.7 \%)$ & \\
\hline Negative & $45(93.8 \%)$ & $7(33.3 \%)$ & \\
\hline CD8 & & & $<0.001$ \\
\hline Positive & $3(6.3 \%)$ & $15(53.6 \%)$ & \\
\hline Negative & $45(93.8 \%)$ & $13(46.4 \%)$ & \\
\hline Venous/lymphatic/Perineural invasion & & & 0.632 \\
\hline No & $18(75.0 \%)$ & $17(81.0 \%)$ & \\
\hline Yes & $6(25.0 \%)$ & $4(19.0 \%)$ & \\
\hline Adjuvant radiotherapy & & & 0.593 \\
\hline No & $21(50.0 \%)$ & $12(57.1 \%)$ & \\
\hline Yes & $21(50.0 \%)$ & $9(42.9 \%)$ & \\
\hline Adjuvant chemotherapy & & & 0.109 \\
\hline No & $49(83.1 \%)$ & $19(67.9 \%)$ & \\
\hline Yes & $10(16.9 \%)$ & $9(32.1 \%)$ & \\
\hline Family history of cancer & & & 0.242 \\
\hline No & $52(88.1 \%)$ & $22(78.6 \%)$ & \\
\hline Yes & $7(11.9 \%)$ & $6(21.4 \%)$ & \\
\hline
\end{tabular}

3.2 Comparing the tumour microenvironment between patients with LC\&TB and LC 
The LC\&TB group included 10 patients with both tumour and TB samples, which were evaluated for differences in terms of PD-L1 expression and TILs (Supplementary Table S2). No significant differences were observed, although the TB lesions tended to have greater $\mathrm{CD} 3^{+}$and $\mathrm{CD} 4^{+} \mathrm{T}$-cell infiltration (vs. the tumours), especially in patients with ATB. However, a comparison of specimens from the LC and LC\&TB groups revealed significant differences in TILs and PD-L1 expression. First, PD-L1 expression was remarkably less common in the LC\&TB group (13 patients, $23.2 \%$ ) than in the LC group (18 patients, 64.3\%). Moreover, the LC\&TB group had clearly lower infiltration of $\mathrm{CD}^{+}, \mathrm{CD}^{+}$, and $\mathrm{CD} 8^{+} \mathrm{T}$-cells, relative to the LC group (all $P<0.001$, Table 1, Supplementary Figures S1-4). Moreover, the predominant phenotype in the LC\&TB group involved no PD-L1 expression and CD8- T-cell infiltration $(P<0.001)$, while the predominant phenotype in the LC group involved PD-L1 expression and CD $8^{+} \mathrm{T}$-cell infiltration (Fig. 2A-C).

\subsection{Survival}

Relative to the LC group, the LC\&TB group had shorter median overall survival (OS: 34.1 months vs, not reached, $P=0.041$, Fig. 2D). Univariate and multivariable survival analyses were performed for the LC\&TB group, although OS was not significantly associated with $\mathrm{CD} 3^{+} / \mathrm{CD} 4^{+} / \mathrm{CD} 8^{+}$T-cell infiltration or PD-L1 expression (Fig. 2E-H). However, OS was significantly associated with pathological T status $(P=0.049)$, lymph node metastasis $(P=0.007)$, and clinical stage $(P=0.006)$ (Fig. 2l, Supplementary Table S3). Cox regression analysis revealed that OS was independently predicted by clinical stage (hazard ratio: 5.70, 95\% confidence interval: $1.26-25.92, P=0.024)$.

\subsection{Genomic alterations}

To reduce the effects of selection bias, propensity score matching analysis was performed to create 21 pairs of patients with LC\&TB or LC alone, who were matched according to sex, age, smoking status, clinical stage, and pathological type (adenocarcinoma or non-adenocarcinoma) (Fig. 1). The samples from these patients were subjected to WES, although 2 samples from the LC\&TB group were excluded because of poor DNA quality. The paired patients' clinicopathological characteristics are shown in Table 2. Figure 3 shows the somatic mutations, clinical features, potential driver genes, 30 most commonly mutated genes, and genes that are related to the efficacy of immune checkpoint inhibitor treatment. 
Table 2

The clinicopathological characteristics of entire patient cohort (divided into two groups).

\begin{tabular}{|c|c|c|c|}
\hline Patient Characteristics & LC with $\mathrm{TB}(\mathrm{N}=19)$ & $L C(N=21)$ & $\begin{array}{l}P \\
\text { value }\end{array}$ \\
\hline Sex & & & 0.906 \\
\hline Male & $13(68.4 \%)$ & $14(66.7 \%)$ & \\
\hline Female & $6(31.6 \%)$ & $7(33.3 \%)$ & \\
\hline Median Age & $54(36-75)$ & $61(37-74)$ & \\
\hline \multicolumn{4}{|l|}{ (Range) } \\
\hline Smoking status & & & 0.583 \\
\hline Non-smoker & $6(31.6 \%)$ & $5(23.8 \%)$ & \\
\hline Smoker & $13(68.4 \%)$ & $16(76.2 \%)$ & \\
\hline Histology & & & 0.105 \\
\hline adenocarcinoma & $13(68.4 \%)$ & $9(42.9 \%)$ & \\
\hline Non-adenocarcinoma & $6(31.6 \%)$ & $12(57.1 \%)$ & \\
\hline Pathologic T stage & & & 0.874 \\
\hline $\mathrm{T} 1+\mathrm{T} 2$ & $15(78.9 \%)$ & $17(81.0 \%)$ & \\
\hline $\mathrm{T} 3+\mathrm{T} 4$ & $4(21.1 \%)$ & $4(19.0 \%)$ & \\
\hline Lymph node metastasis & & & 0.385 \\
\hline NO & 15 (78.9\%) & $14(66.7 \%)$ & \\
\hline N1-3 & $4(21.1 \%)$ & $7(33.3 \%)$ & \\
\hline Distant metastasis & & & 0.287 \\
\hline MO & $18(94.7 \%)$ & $21(100.0 \%)$ & \\
\hline M1 & $1(5.3 \%)$ & $0(0 \%)$ & \\
\hline Clinical stage & & & 0.583 \\
\hline I+ II & $13(68.4 \%)$ & $16(76.2 \%)$ & \\
\hline III & $6(31.6 \%)$ & $5(23.8 \%)$ & \\
\hline Differentiation & & & 0.588 \\
\hline Poor or undifferentiated & $9(56.3 \%)$ & $6(46.2 \%)$ & \\
\hline Well-intermediate & $7(43.8 \%)$ & $7(53.8 \%)$ & \\
\hline
\end{tabular}




\begin{tabular}{|c|c|c|c|}
\hline Patient Characteristics & LC with TB(N = 19) & $\mathrm{LC}(\mathrm{N}=21)$ & $\begin{array}{l}P \\
\text { value }\end{array}$ \\
\hline $\begin{array}{l}\text { Venous/lymphatic/Perineural } \\
\text { invasion }\end{array}$ & & & 0.807 \\
\hline No & $14(77.8 \%)$ & $17(81.0 \%)$ & \\
\hline Yes & $4(22.2 \%)$ & $4(19.0 \%)$ & \\
\hline Adjuvant radiotherapy & & & 0.573 \\
\hline No & $8(53.3 \%)$ & $6(42.9 \%)$ & \\
\hline Yes & $7(46.7 \%)$ & $8(57.1 \%)$ & \\
\hline Adjuvant chemotherapy & & & 0.583 \\
\hline No & 15 (78.9\%) & $15(71.4 \%)$ & \\
\hline Yes & $4(21.1 \%)$ & $6(28.6 \%)$ & \\
\hline Family history of cancer & & & 0.916 \\
\hline No & 17 (89.5\%) & 19 (90.5\%) & \\
\hline Yes & $2(10.5 \%)$ & $2(9.5 \%)$ & \\
\hline EGFR & & & 0.101 \\
\hline Positive & $1(5.3 \%)$ & $5(23.8 \%)$ & \\
\hline Negative & $18(94.7 \%)$ & $16(76.2 \%)$ & \\
\hline PD-L1 & & & 0.005 \\
\hline Positive & $2(10.5 \%)$ & $11(52.4 \%)$ & \\
\hline Negative & 17 (89.5\%) & $10(47.6 \%)$ & \\
\hline CD3 & & & 0.163 \\
\hline Positive & $4(28.6 \%)$ & $11(52.4 \%)$ & \\
\hline Negative & $10(71.4 \%)$ & $10(47.6 \%)$ & \\
\hline CD4 & & & 0.009 \\
\hline Positive & $3(21.4 \%)$ & $14(66.7 \%)$ & \\
\hline Negative & $11(78.6 \%)$ & $7(33.3 \%)$ & \\
\hline CD8 & & & 0.045 \\
\hline Positive & $1(7.1 \%)$ & $8(38.1 \%)$ & \\
\hline Negative & 13 (92.7\%) & $13(61.9 \%)$ & \\
\hline
\end{tabular}




\begin{tabular}{|llll|}
\hline Patient Characteristics & LC with TB(N=19) & LC (N= 21) & $\begin{array}{c}P \\
\text { value }\end{array}$ \\
\hline TP53 & & & 0.012 \\
\hline Positive & $7(36.8 \%)$ & $16(76.2 \%)$ & \\
\hline Negative & $12(63.2 \%)$ & $5(23.8 \%)$ & 0.042 \\
\hline C1QB & & & \\
\hline Positive & $4(21.1 \%)$ & $21(100 \%)$ & 0.916 \\
\hline Negative & $15(63.2 \%)$ & & 0.141 \\
\hline CDKN2A & & $2(9.5 \%)$ & \\
\hline Positive & $2(10.5 \%)$ & $19(90.5 \%)$ & \\
\hline Negative & $17(89.5 \%)$ & & \\
\hline PoU3F3 & & $1(4.8 \%)$ & \\
\hline Positive & $4(21.1 \%)$ & $20(95.2 \%)$ & $10.8(2.0-25.1)$ \\
\hline Negative & $15(78.9 \%)$ & muts/MB & \\
\hline Median TMB (Range) & $17.4(7.5-30.6)$ & muts/MB & \\
\hline
\end{tabular}

The most common alterations in the LC\&TB group were mutations in ZNF208 (74\%), FLG (68\%), MUC17 (68\%), ZNF729 (63\%), and HRNR (58\%) (Supplementary Figure S2A). The most common alterations in the LC group were mutations in TP53 (76\%), TTN (67\%), RYR2 (43\%), KMT2D (38\%), and MUC4 (38\%). The differential analysis results for somatic mutations in the LC\&TB and LC groups are listed in Supplementary Table S4. There were no significant differences between the LC\&TB and LC groups in terms of somatic mutations in EGFR, PIK3CA, KRAS, and BRAF, as well as CDKN2A copy number variations (CNVs) (all $P>0.05$ ), (Supplementary Figure S6). However, a significant difference in the average number of single-nucleotide variants/insertions/deletions was observed between the LC and LC\&TB groups $(P=0.002)$, as well as between the LC\&ATB $(n=1,131.08)$, LC\&O/CTB $(n=1,143.83)$, and LC $(n=633.95)$ groups $(P<0.001)$ (Fig. 4A). The most common base substitution was $C>T$ in all three groups, with frequencies of $34.4 \%$ in the LC\&ATB group, $35.41 \%$ in the LC\&O/CTB group, and $35.27 \%$ in the LC group, although the differences were not statistically significant (all $P>0.05$ ) (Fig. 4B). The average number of CNVs per sample were 41.84 in the LC\&ATB group, 38.67 in the LC\&O/CTB group, and 36.67 in the LC group, although the differences were not statistically significant (Fig. 4C). A heatmap of the gain/loss CNVs is shown in Supplementary Figure S7A and a heatmap of the 50 most commonly affected genes in the LC\&ATB and LC groups is shown in Supplementary Figure S7B. There were also no significant differences in the numbers of CNVs in the CD274 and CDKN2A genes (Supplementary Figure $\mathrm{S6H})$. The results of the GO and KEGG pathway analyses are shown in Supplementary Figure S7C-D, which revealed that the top functional clusters in the LC\&TB group involved cancer-related pathways. 
Mutation signatures were first described in 2013 (23) as groups of gene mutations that are related to malignant processes in tumour cells. We performed mutation signature analyses for each group, which revealed mutation signature 4 in the LC\&TB and LC groups. Cluster analysis also revealed that the proportion of samples with mutation signature 1 was higher in the LC\&TB group than in the LC group (32.99\% vs. $11.23 \%$ ) (Supplementary Figure S8A-C). Mutually exclusive and co-occurring gene pairs are shown in Supplementary Figure S8D.

\subsection{TMB analysis, HLA analysis, and neoantigen prediction}

The LC\&ATB and LC\&O/CTB groups were characterized by a high average TMB (18.65 mutations/Mb and 18.86 mutations/Mb), which was noticeably higher than the average TMB in the LC group (10.45 mutations/Mb) (Fig. 5A). Furthermore, a high TMB was significantly associated with a mutation signature that suggested exposure to cigarette smoke in the LC group $(P=0.036)$ and the LC\&TB group $(P=0.025)$ (Fig. 5C). Interestingly, a lower TMB was observed in LC\&TB patients with a low TP53 mutation frequency $(P=0.027$, Fig. $5 \mathrm{D})$. The average numbers of neoantigens were $1,146.08$ in the LC\&ATB group, 888.33 in the LC\&O/CTB group, and 395.29 in the LC group $(P<0.001$, Fig. 5E), and the number of nonsynonymous mutations was correlated with the number of neoantigens $(r=0.74, P<0.01$, Fig. 5G). Expression of PD-L1 was not significantly correlated with the TMB among all patients with WES results $(r=0.71, P=0.258$, Fig. $5 \mathrm{H}$ ). The three most common HLA types were identified in each group, and the results revealed heterozygous genotypes for all HLA class I subtypes (A, B, and C) in the LC\&ATB, LC\&O/CTB, and LC groups ( $P>0.05$, Supplementary Table S5).

We also found several potential driver genes in patients with LC\&TB (Supplementary Materials).

\section{Discussions}

Preclinical studies have indicated that immune homeostasis in TB is regulated via immune checkpoint pathways, such as the PD-1/PD-L1 axis. For example, in animal models, the PD-1 pathway plays a key role in controlling excessive inflammation after MTB infection and regulating the resulting immune response (24). Furthermore, the PD-1/PD-L1 pathway suppresses the accumulation of CD $4{ }^{+}$T-cells and IFN-y production, which is an essential part of the immune response to TB. However, treatment that targets PD-1/PD-L1 may cause CD4 + T-cells to overproduce IFN- $\gamma$, which can aggravate TB or cause TB recurrence (24-35). Another study has indicated that a TB antigen can inhibit the Th1 immune response and promote LC metastasis via the PD-1/PD-L1 signalling pathway (36). However, there are limited data regarding the immune microenvironment in patients with $L C$ and previous/current $T B$, which highlights the need for additional information regarding PD-L1 expression and TILs in the tumour and surrounding microenvironment. Our findings revealed that, relative to the LC group, the LC\&TB group had significantly decreased PD-L1 expression and less $\mathrm{CD}^{+} / \mathrm{CD}^{+} / \mathrm{CD} 8^{+} \mathrm{T}$-cell infiltration, which suggests that these patients have immunologically cold tumours in a noninflammatory microenvironment (Fig. 6A). To the best of our knowledge, this is the first comprehensive analysis of the tumour microenvironment landscape in LC\&TB and LC. 
Previous studies have suggested that PD-L1 expression, TP53 mutation frequency, TMB (37), and HLA molecules may be biomarkers for predicting the response of LC to anti-PD-1/PD-L1 therapy (38-40), although these relationships remain controversial. In our study, the LC\&TB group had a markedly higher TMB than the LC group, and the LC\&TB group also had a significantly lower TP53 mutation frequency. The present study revealed that the LC\&TB and LC groups only had heterozygous HLA I genotype, and thus we did not perform any additional analyses. Interestingly, we also observed that the number of nonsynonymous mutations was correlated with the number of neoantigens, which is consistent with previous research (41). Moreover, the TMB was significantly associated with smoking history among patients with LC\&TB and LC alone, which also agrees with previously reported results (42). These results suggest that there are significant differences in terms of genomic alterations and mutation signatures between the LC\&TB and LC groups.

A few studies have attempted to identify potential driver genes in patients with LC who had a history of TB $(43,44)$. Adenocarcinoma was the main pathological type of LC among our patients with LC\&TB, which is consistent with previously reported results (44). Although, patients with lung adenocarcinoma who have a history of lung scarring or TB are more likely to develop EGFR mutations, relative to patients with conventional lung cancer (44). Nevertheless, the present study failed to detect a significant difference between the LC\&TB and LC groups in terms of the EGFR mutation frequencies.

Notably, there were potential gene mutations differences between ATB and O/CTB in present study. For instance, The $C 1 Q B$ gene encodes the B-chain polypeptide of complement subcomponent C1q. The complement pathway is an important part of the immune system, and complement-mediated bacteriolysis and cytolysis are important mechanisms in the response to infection by pathogenic microorganisms. We observed that it was mutated at a higher rate among patients with LC\&TB (5/19 cases) than among patients with LC alone (0/21 cases). In addition, bioinformatics analysis predicted that $C 1 Q B$ c.274_311del would have a substantial effect on protein expression. Furthermore, the $C 1 Q B$ gene may influence macrophage and dendritic cell infiltration of NSCLC. Therefore, C1QB may be a driver gene that leads to the developing of LC in patients with lung scarring or TB. In addition, KRAS mutation differences are highly significant between ATB and O/CTB ( $P=0.003$ in Supplementary Figure S6D), but when combined as a single group, there is no difference between LT\&TB and LC alone. However, due to the limited sample size of these two groups, it is difficult to draw a clear conclusion. Further studies with large sample size and mechanism investigation are urgently needed.

Because a noninflammatory microenvironment we have observed in LC\&TB and the possibility of reactivation/exacerbation of TB by anti-PD-1/PD-L1 therapy, which suggests that there is a need for new approaches to treating patients with LC\&TB. We propose three potential strategies for treating patients with LC\&TB (Fig. 6B). First, chimeric antigen receptor T-cell immunotherapy strategies that directly target $\mathrm{CD}^{+}$T-cells might help avoid overactivation of $\mathrm{CD} 4^{+}$T-cells. Second, dendritic cell vaccines could be developed for patients with LC\&TB. Third, it might be useful to consider the possibility of drugs that target mutated $C 1 Q B$. 


\section{Conclusions}

In conclusion, we identified significant differences in the tumour microenvironment and genomic alterations when we compared patients with LC\&TB and LC alone. In particular, patients with LC\&TB had significantly lower PD-L1 expression and lower infiltration of immune cells. Moreover, patients with LC\&TB had a lower TP53 mutation frequency, as well as higher values for TMB, number of neoantigens, and frequency of mutation signature 1. Moreover, our findings suggest that $C 1 Q B$ may be a driver gene in patients with LC\&TB, and further studies are needed to investigate the specific contributions of $C 1 Q B$ mutations in this setting.

\section{Abbreviations}

ATB: active tuberculosis; C1QB: completement C1q B chain; CNVs: copy number variations; FFPE: formalin-fixed paraffin embedded; LC\&TB: lung cancer and tuberculosis; LC: lung cancer; MTB: Mycobacterium tuberculosis; NSCLC: non-small cell lung cancer; OS: overall survival; TB: tuberculosis; OTB: obsolete tuberculosis; ATB: active tuberculosis; CTB: Cured tuberculosis; O/CTB: obsolete/cured tuberculosis; IGRA: interferon gamma release assay; TIL: tumour-infiltrating lymphocytes; TMB: tumour mutation burden; TPS: tumour proportion score; WES: whole-exome sequencing.

\section{Declarations}

\section{Ethics approval and consent to participate}

The studies involving human participants were reviewed and approved by the Ethics Committee of the Zhejiang Cancer Hospital and Affiliated Hangzhou Chest Hospital. Written informed consent for participation was not required for this study in accordance with the national legislation and the institutional requirements.

\section{Consent for publication}

Not applicable.

\section{Availability of data and material}

Data supporting the results of this study are available from the corresponding author.

\section{Competing interests}

No potential conflicts of interest were reported by the authors.

\section{Funding}

This work was supported by grants from the National Natural Science Foundation of China [81802995 and81672315], CSCO foundation [Y-Q201802-074], the Zhejiang Province Public Welfare Funds [LGF19H280004].

\section{Authors' contributions}


YF and WMM designed and supervised the project. TXW CHB XH and YY performed the experiments described in the manuscript. CHB and TXW analyzed the data. XLX and TXW wrote the original manuscript. YF CCW and DC gave approval for the final version of the manuscript. All authors read and approved the final manuscript.

\section{Acknowledgements}

We thank the approve from the Zhejiang Cancer Hospital and Affiliated Hangzhou Chest Hospital in this study.

\section{References}

1. Torre LA, Siegel RL, Jemal A. Lung Cancer Statistics. Adv Exp Med Biol. 2016;893:1-19.

2. Chen W, Zheng R, Zhang S, Zeng H, Xia C, Zuo T, et al. Cancer incidence and mortality in China, 2013. Cancer Lett. 2017;401:63-71.

3. Hong S, Mok Y, Jeon C, Jee SH, Samet JM. Tuberculosis, smoking and risk for lung cancer incidence and mortality. Int J Cancer. 2016;139(11):2447-55.

4. Shen X, Wang L, Zhu L. Spatial Analysis of Regional Factors and Lung Cancer Mortality in China, 1973-2013. Cancer Epidemiol Biomarkers Prev. 2017;26(4):569-77.

5. Walzl G, McNerney R, du Plessis N, Bates M, McHugh TD, Chegou NN, et al. Tuberculosis: advances and challenges in development of new diagnostics and biomarkers. Lancet Infect Dis. 2018;18(7):e199-e210.

6. Bhatt M, Kant S, Bhaskar R. Pulmonary tuberculosis as differential diagnosis of lung cancer. South Asian J Cancer. 2012;1(1):36-42.

7. Keikha M, Esfahani BN. The Relationship between Tuberculosis and Lung Cancer. Adv Biomed Res. 2018;7:58.

8. Simsek A, Kalemci S, Mutlu N, Yapici I, Acet Ozturk NA. Lung cancer diagnosed with Mycobacterium tuberculosis or nontuberculosis mycobacteria concomitantly. Tuberk Toraks. 2017;65(4):291-5.

9. Wong JYY, Zhang H, Hsiung CA, Shiraishi K, Yu K, Matsuo K, et al. Tuberculosis infection and lung adenocarcinoma: Mendelian randomization and pathway analysis of genome-wide association study data from never-smoking Asian women. Genomics. 2020;112(2):1223-32.

10. Lee HY, Kim JW, Yeo CD. A case of tuberculosis reactivation suspected of cancer progression during oral tyrosine kinase inhibitor treatment in a patient diagnosed as non-small cell lung cancer. $J$ Thorac Dis. 2017;9(8):E709-E13.

11. Evman S, Baysungur V, Alpay L, Uskul B, Misirlioglu AK, Kanbur S, et al. Management and Surgical Outcomes of Concurrent Tuberculosis and Lung Cancer. Thorac Cardiovasc Surg. 2017;65(7):542-5.

12. Rittmeyer A, Barlesi F, Waterkamp D, Park K, Ciardiello F, von Pawel J, et al. Atezolizumab versus docetaxel in patients with previously treated non-small-cell lung cancer (OAK): a phase 3, open-label, multicentre randomised controlled trial. Lancet. 2017;389(10066):255-65. 
13. Kato K, Cho BC, Takahashi M, Okada M, Lin CY, Chin K, et al. Nivolumab versus chemotherapy in patients with advanced oesophageal squamous cell carcinoma refractory or intolerant to previous chemotherapy (ATTRACTION-3): a multicentre, randomised, open-label, phase 3 trial. Lancet Oncol. 2019;20(11):1506-17.

14. Fehrenbacher L, von Pawel J, Park K, Rittmeyer A, Gandara DR, Ponce Aix S, et al. Updated Efficacy Analysis Including Secondary Population Results for OAK: A Randomized Phase III Study of Atezolizumab versus Docetaxel in Patients with Previously Treated Advanced Non-Small Cell Lung Cancer. J Thorac Oncol. 2018;13(8):1156-70.

15. Borghaei H, Paz-Ares L, Horn L, Spigel DR, Steins M, Ready NE, et al. Nivolumab versus Docetaxel in Advanced Nonsquamous Non-Small-Cell Lung Cancer. N Engl J Med. 2015;373(17):1627-39.

16. Brahmer J, Reckamp KL, Baas P, Crino L, Eberhardt WE, Poddubskaya E, et al. Nivolumab versus Docetaxel in Advanced Squamous-Cell Non-Small-Cell Lung Cancer. N Engl J Med. 2015;373(2):12335.

17. Ready N, Farago AF, de Braud F, Atmaca A, Hellmann MD, Schneider JG, et al. Third-Line Nivolumab Monotherapy in Recurrent SCLC: CheckMate 032. J Thorac Oncol. 2019;14(2):237-44.

18. Chan GH, Gwee YX, Low JL, Huang Y, Chan ZY, Choo JR, et al. Immune checkpoint inhibition for nonsmall cell lung cancer in patients with pulmonary tuberculosis or Hepatitis B: Experience from a single Asian centre. Lung Cancer. 2020;146:145-53.

19. Barber DL, Mayer-Barber KD, Feng CG, Sharpe AH, Sher A. CD4 T cells promote rather than control tuberculosis in the absence of PD-1-mediated inhibition. J Immunol. 2011;186(3):1598-607.

20. Gonzalez-Cao M, Puertolas T, Riveiro M, Munoz-Couselo E, Ortiz C, Paredes R, et al. Cancer immunotherapy in special challenging populations: recommendations of the Advisory Committee of Spanish Melanoma Group (GEM). J Immunother Cancer. 2021;9(3).

21. Chen S, Zhou Y, Chen Y, Gu J. fastp: an ultra-fast all-in-one FASTQ preprocessor. Bioinformatics. 2018;34(17):i884-i90.

22. Yu H, Chen Z, Ballman KV, Watson MA, Govindan R, Lanc I, et al. Correlation of PD-L1 Expression with Tumor Mutation Burden and Gene Signatures for Prognosis in Early-Stage Squamous Cell Lung Carcinoma. J Thorac Oncol. 2019;14(1):25-36.

23. Alexandrov LB, Nik-Zainal S, Wedge DC, Aparicio SA, Behjati S, Biankin AV, et al. Signatures of mutational processes in human cancer. Nature. 2013;500(7463):415-21.

24. Zaemes J, Kim C. Immune checkpoint inhibitor use and tuberculosis: a systematic review of the literature. Eur J Cancer. 2020;132:168-75.

25. Adekambi T, Ibegbu CC, Kalokhe AS, Yu T, Ray SM, Rengarajan J. Distinct effector memory CD4+ T cell signatures in latent Mycobacterium tuberculosis infection, BCG vaccination and clinically resolved tuberculosis. PLoS One. 2012;7(4):e36046.

26. Anastasopoulou A, Ziogas DC, Samarkos M, Kirkwood JM, Gogas H. Reactivation of tuberculosis in cancer patients following administration of immune checkpoint inhibitors: current evidence and clinical practice recommendations. J Immunother Cancer. 2019;7(1):239. 
27. Byeon S, Cho JH, Jung HA, Sun JM, Lee SH, Ahn JS, et al. PD-1 inhibitors for non-small cell lung cancer patients with special issues: Real-world evidence. Cancer Med. 2020;9(7):2352-62.

28. Chu YC, Fang KC, Chen HC, Yeh YC, Tseng CE, Chou TY, et al. Pericardial Tamponade Caused by a Hypersensitivity Response to Tuberculosis Reactivation after Anti-PD-1 Treatment in a Patient with Advanced Pulmonary Adenocarcinoma. J Thorac Oncol. 2017;12(8):e111-e4.

29. Lee JJ, Chan A, Tang T. Tuberculosis reactivation in a patient receiving anti-programmed death-1 (PD-1) inhibitor for relapsed Hodgkin's lymphoma. Acta Oncol. 2016;55(4):519-20.

30. Picchi H, Mateus C, Chouaid C, Besse B, Marabelle A, Michot JM, et al. Infectious complications associated with the use of immune checkpoint inhibitors in oncology: reactivation of tuberculosis after anti PD-1 treatment. Clin Microbiol Infect. 2018;24(3):216-8.

31. Tezera LB, Bielecka MK, Ogongo P, Walker NF, Ellis M, Garay-Baquero DJ, et al. Anti-PD-1 immunotherapy leads to tuberculosis reactivation via dysregulation of TNF-a. Elife. 2020;9.

32. Tsai CC, Chen JH, Wang YC, Chang FY. Re-activation of pulmonary tuberculosis during antiprogrammed death-1 (PD-1) treatment. QJM. 2019;112(1):41-2.

33. van Eeden R, Rapoport BL, Smit T, Anderson R. Tuberculosis Infection in a Patient Treated With Nivolumab for Non-small Cell Lung Cancer: Case Report and Literature Review. Front Oncol. 2019;9:659.

34. Kyi C, Hellmann MD, Wolchok JD, Chapman PB, Postow MA. Opportunistic infections in patients treated with immunotherapy for cancer. J Immunother Cancer. 2014;2:19.

35. Fujita K, Terashima T, Mio T. Anti-PD1 Antibody Treatment and the Development of Acute Pulmonary Tuberculosis. J Thorac Oncol. 2016;11(12):2238-40.

36. Cao S, Li J, Lu J, Zhong R, Zhong H. Mycobacterium tuberculosis antigens repress Th1 immune response suppression and promotes lung cancer metastasis through PD-1/PDI-1 signaling pathway. Cell Death Dis. 2019;10(2):44.

37. Qian J, Nie W, Lu J, Zhang L, Zhang Y, Zhang B, et al. Racial differences in characteristics and prognoses between Asian and white patients with nonsmall cell lung cancer receiving atezolizumab: An ancillary analysis of the POPLAR and OAK studies. Int J Cancer. 2020;146(11):3124-33.

38. Hurkmans DP, Kuipers ME, Smit J, van Marion R, Mathijssen RHJ, Postmus PE, et al. Tumor mutational load, CD8(+) T cells, expression of PD-L1 and HLA class I to guide immunotherapy decisions in NSCLC patients. Cancer Immunol Immunother. 2020;69(5):771-7.

39. Negrao MV, Lam VK, Reuben A, Rubin ML, Landry LL, Roarty EB, et al. PD-L1 Expression, Tumor Mutational Burden, and Cancer Gene Mutations Are Stronger Predictors of Benefit from Immune Checkpoint Blockade than HLA Class I Genotype in Non-Small Cell Lung Cancer. J Thorac Oncol. 2019;14(6):1021-31.

40. Santaniello A, Napolitano F, Servetto A, De Placido P, Silvestris N, Bianco C, et al. Tumour Microenvironment and Immune Evasion in EGFR Addicted NSCLC: Hurdles and Possibilities. Cancers (Basel). 2019;11(10). 
41. Turajlic S, Litchfield K, Xu H, Rosenthal R, McGranahan N, Reading JL, et al. Insertion-and-deletionderived tumour-specific neoantigens and the immunogenic phenotype: a pan-cancer analysis. Lancet Oncol. 2017;18(8):1009-21.

42. Chae YK, Davis AA, Raparia K, Agte S, Pan A, Mohindra N, et al. Association of Tumor Mutational Burden With DNA Repair Mutations and Response to Anti-PD-1/PD-L1 Therapy in Non-Small-Cell Lung Cancer. Clin Lung Cancer. 2019;20(2):88-96 e6.

43. Luo YH, Wu CH, Wu WS, Huang CY, Su WJ, Tsai CM, et al. Association between tumor epidermal growth factor receptor mutation and pulmonary tuberculosis in patients with adenocarcinoma of the lungs. J Thorac Oncol. 2012;7(2):299-305.

44. Hwang IK, Paik SS, Lee SH. Impact of Pulmonary Tuberculosis on the EGFR Mutational Status and Clinical Outcome in Patients with Lung Adenocarcinoma. Cancer Res Treat. 2019;51(1):158-68.

\section{Figures}




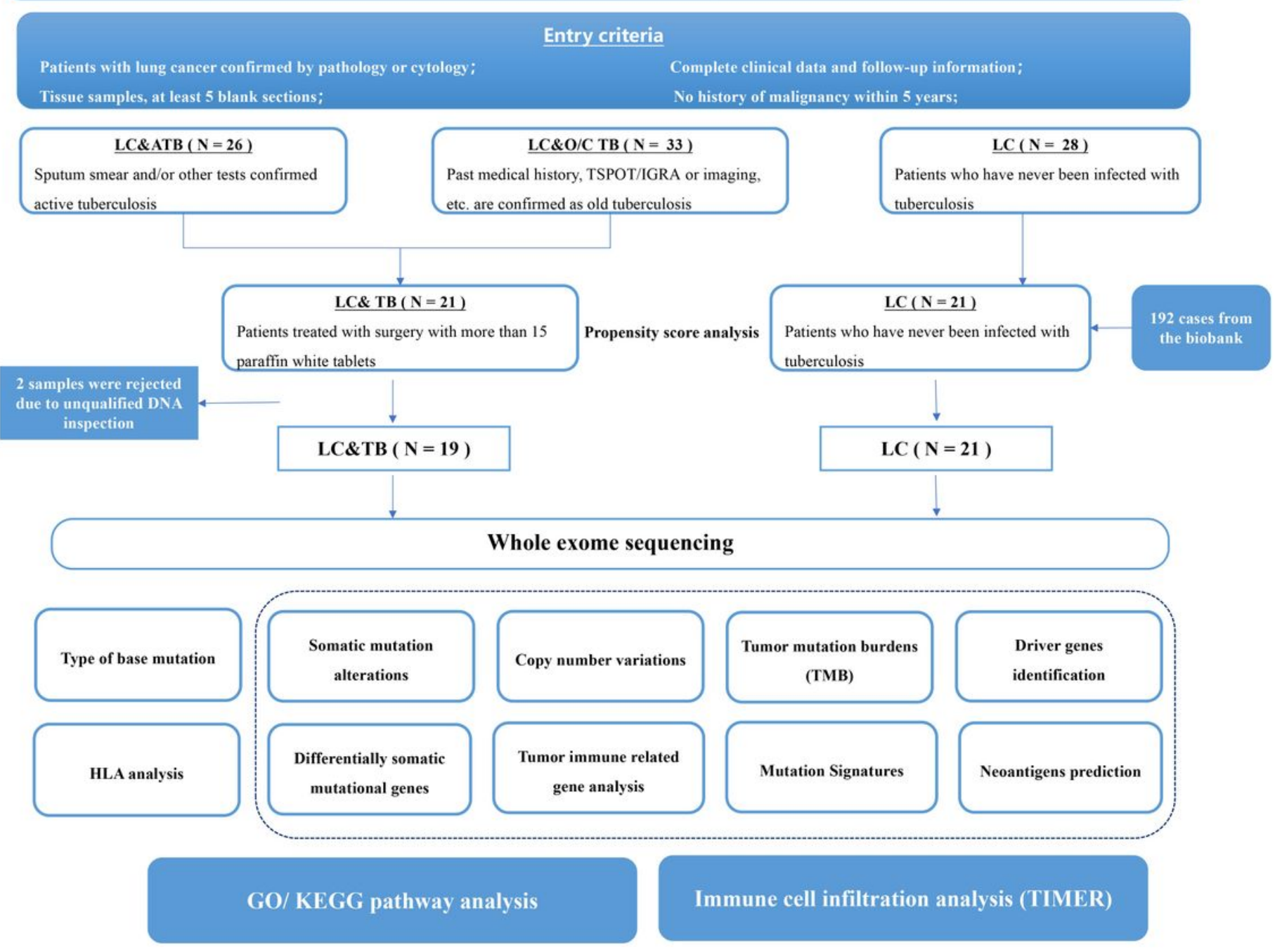

Note: ${ }^{*} 10$ patients are both had TB and Tumor tissue; All analysis in the dashed box had positive findings.

\section{Figure 1}

A flowchart of the clinical and immune microenvironment analyses of 87 samples and the whole-exome sequencing analysis of 40 samples from patients with LC or LC\&ATB. 
A

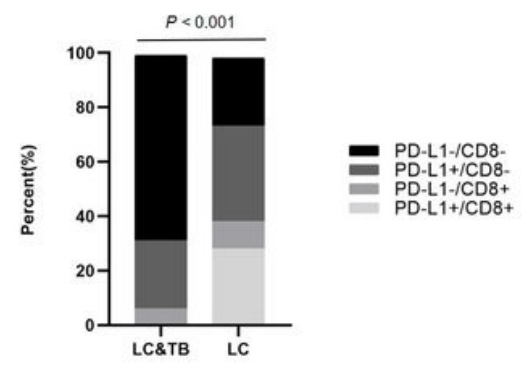

D

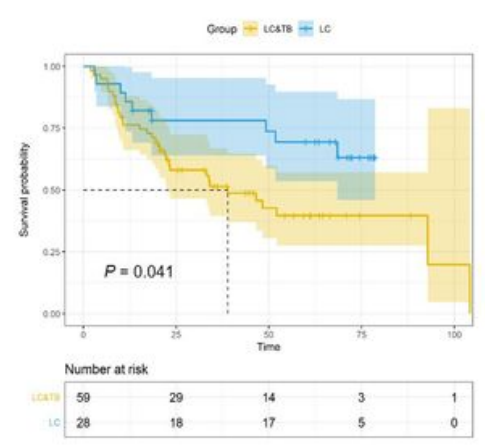

B

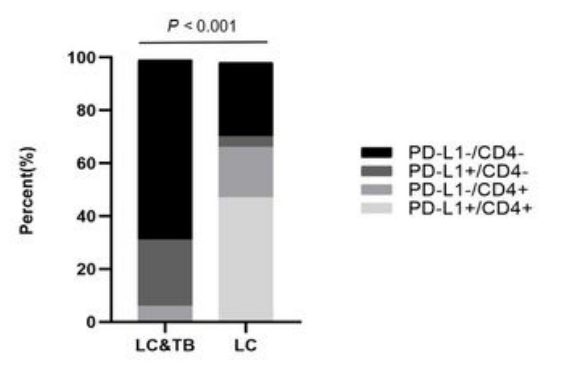

E

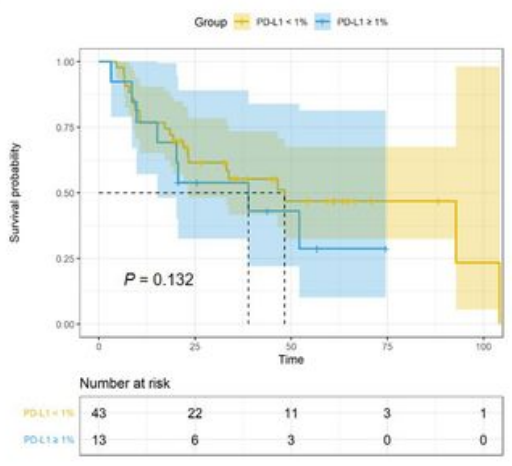

C

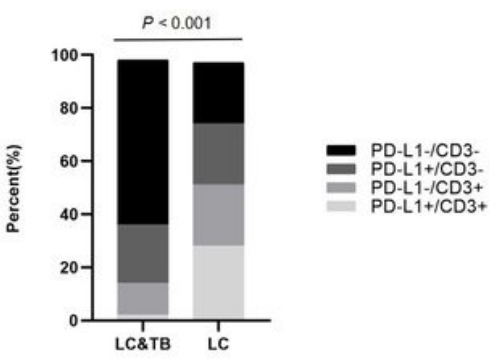

$\mathbf{F}$
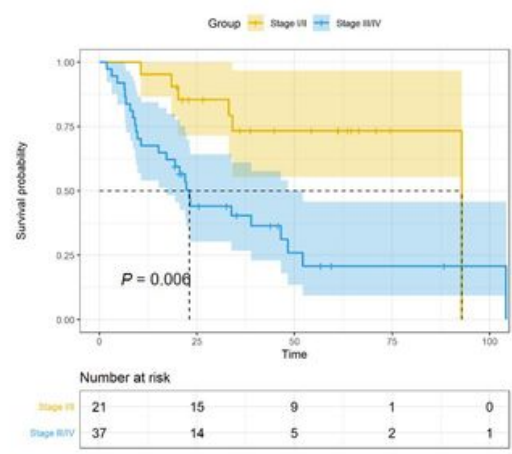

Figure 2

The correlations between tumour immune microenvironment factors and overall survival. Patients with LC alone or LC\&TB were compared in terms of PD-L1 and CD8 expression (A), PD-L1 and CD4 expression (B), and PD-L1 and CD3 expression (C). (D) Kaplan-Meier curves for the LC and LC\&TB groups revealed that the LC group had significantly better survival $(P=0.041)$. $(E)$ Kaplan-Meier curves according to PD-L1 expression revealed no significant differences in overall survival among patients with LC\&TB or LC $(P=$ 0.132). (F) Kaplan-Meier curves according to disease stage revealed that stage III/IV disease was associated with significantly better survival than stage $\mathrm{I} / \mathrm{II}$ disease $)(P=0.006)$. 
A

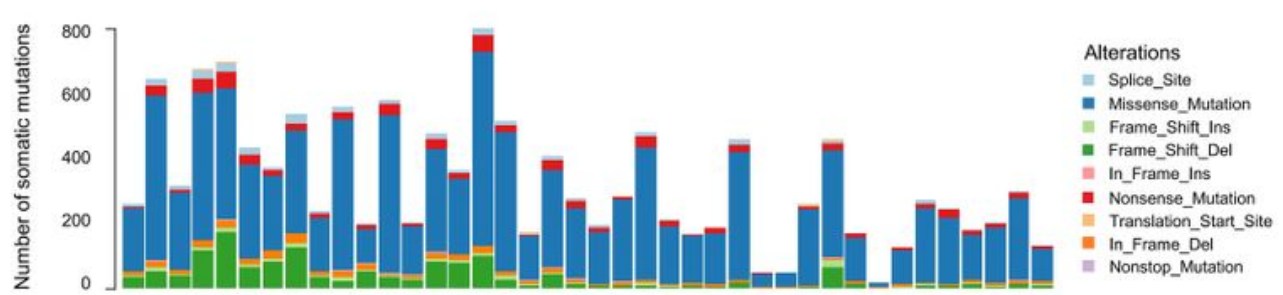

B
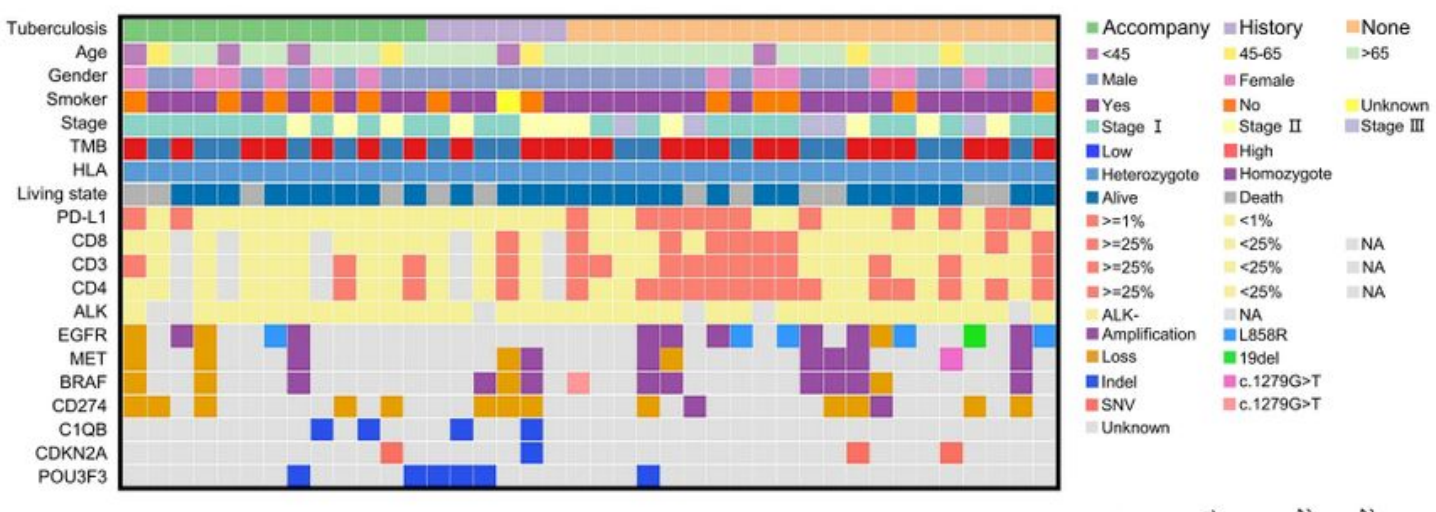

C

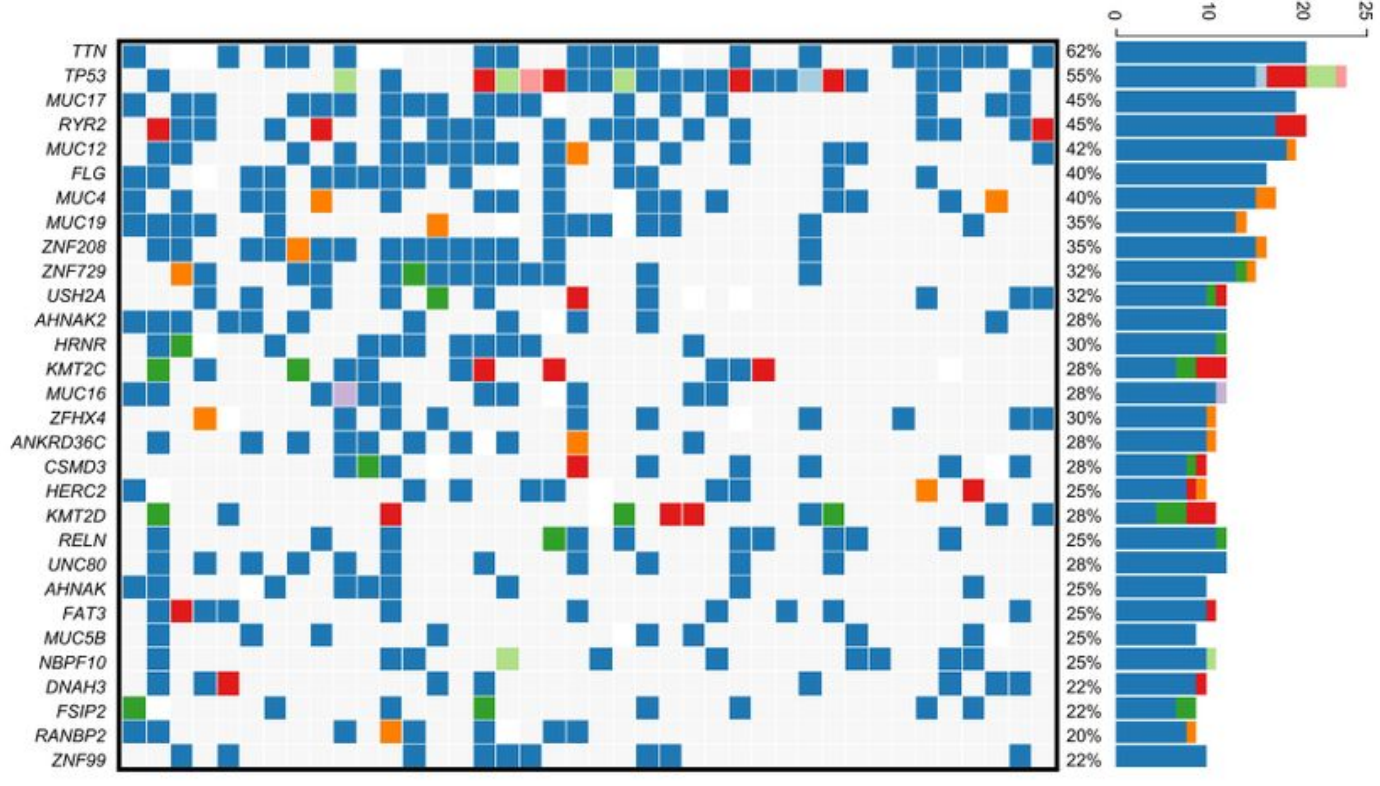

D

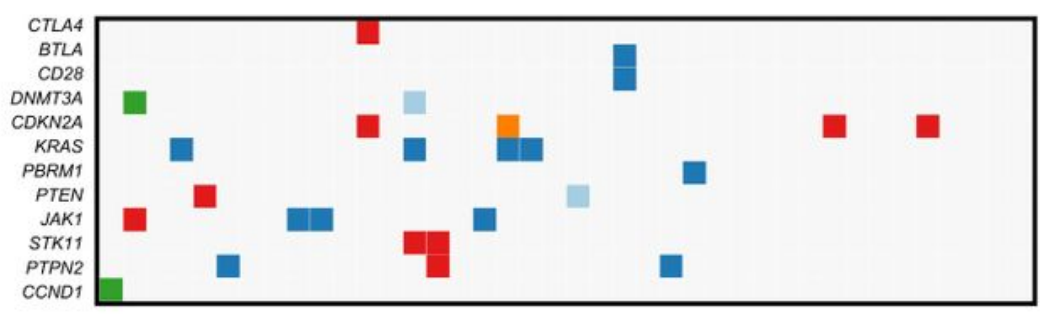

\section{Figure 3}

Summary of the clinical and molecular features associated with LC\&TB or LC. (A) Bar plots showing the numbers of somatic mutations in each patient. (B) Pathology type, age, sex, smoking status, stage, TMB, HLA type, living status, marker expressions (PD-L1, CD3, CD4, and CD8), and somatic mutations for all patients. (C) Heatmap showing the distribution of significantly mutated genes in 40 patients with LC 
(gene mutation frequencies are shown on the right). (D) Heatmap showing the distribution of mutations in immune-related genes from the 40 patients with LC.

A

Single nucleotide variants/Indels
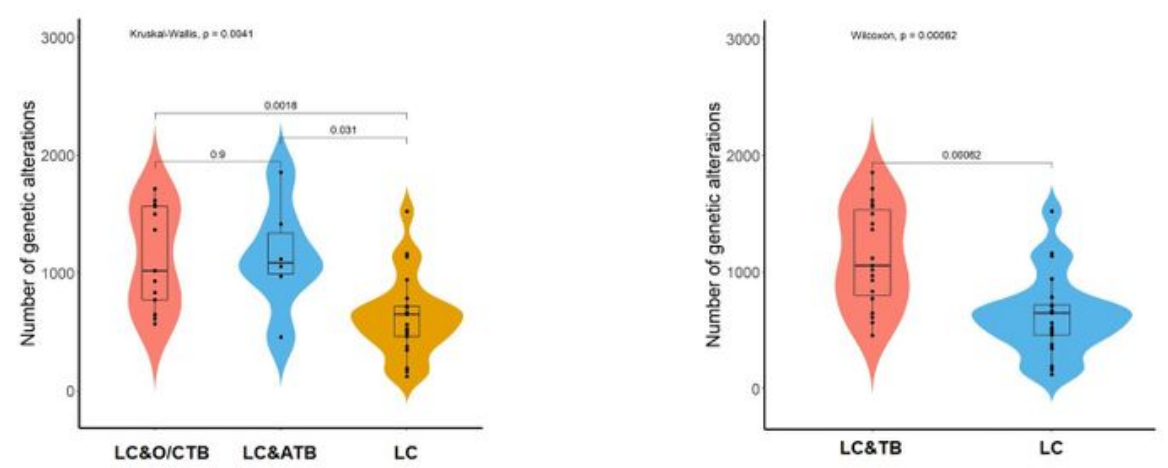

B

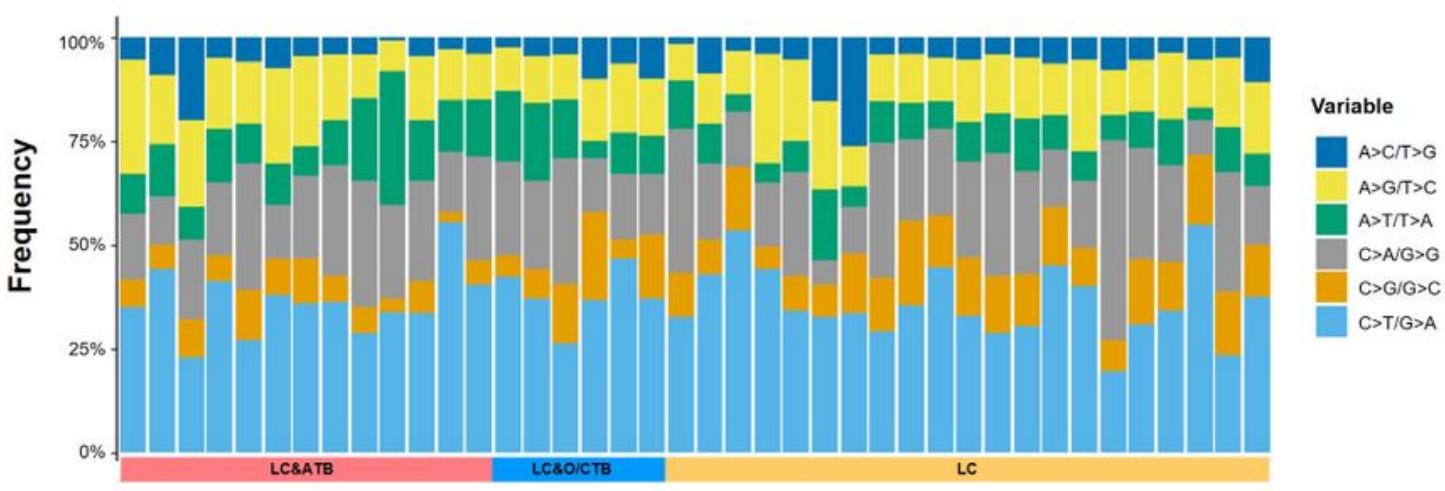

c
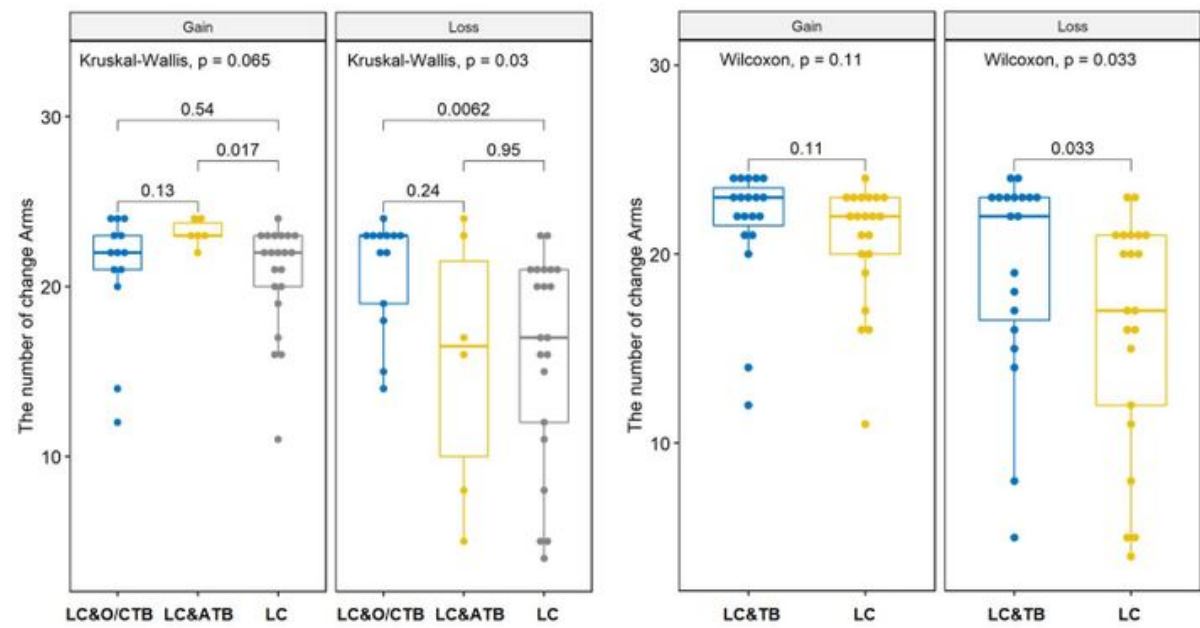

Figure 4

The number of genetic alterations, types of substitution mutations, and the number of gains/losses (CNVs) in patients with LC\&O/CTB, LC\&ATB, or LC. (A) Violin plots showing the numbers of genetic alterations among patients with LC\&O/CTB, LC\&ATB, or LC. (B) Frequencies of mutation substitution 
types (classified as 6 substitution classes) in the genome among all samples. Vertical bars indicate individual patients and the vertical axis shows the frequency of each mutation category for a specific mutation type. Most patients with LC had predominantly $C>T / G>A$ transitions. (C) Boxplots showing the number of gains/losses (CNVs) in each patient from the LC\&O/CTB, LC\&ATB, and LC groups. Significant inter-group differences in the number of losses per patient were detected using the two-sided Wilcoxon rank-sum test.

A

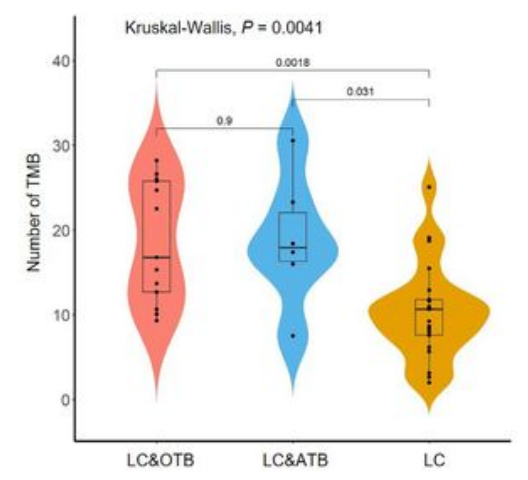

C

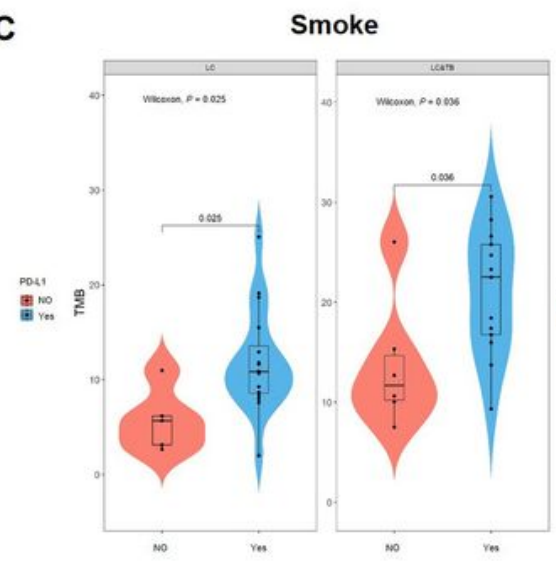

G

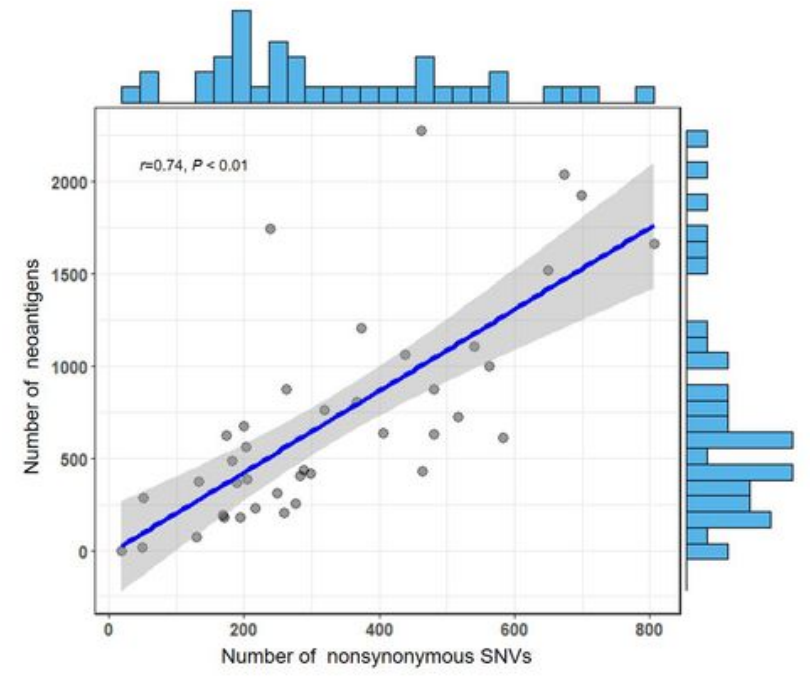

B

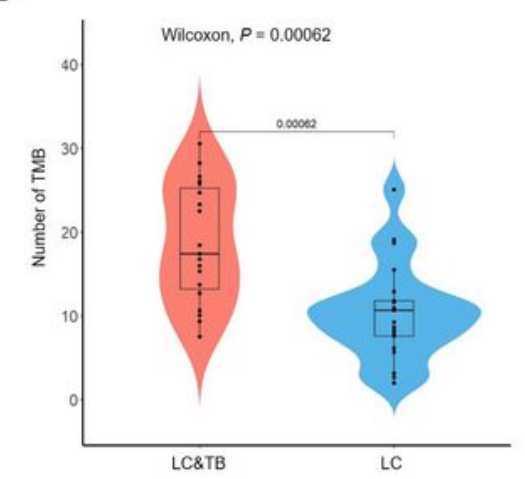

D

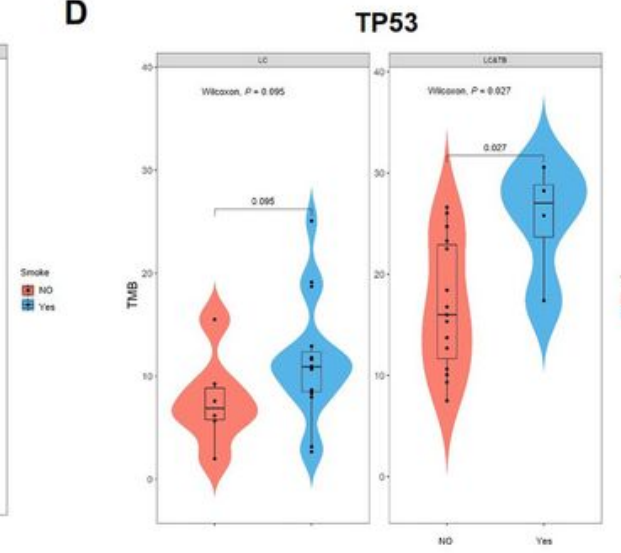

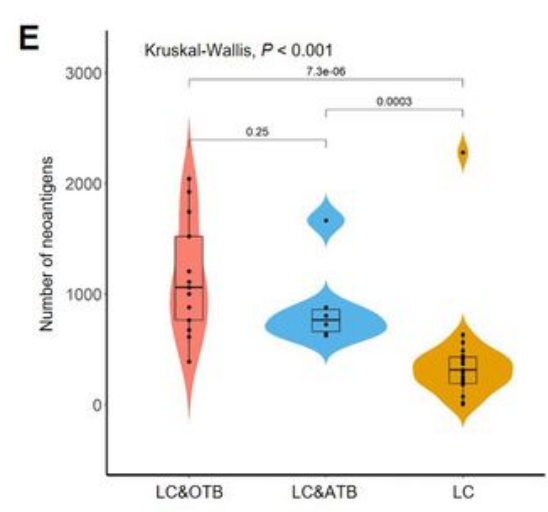

F

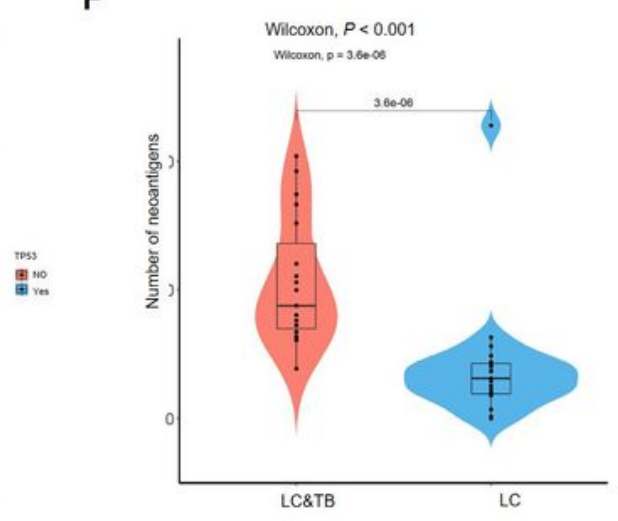

Figure 5

H

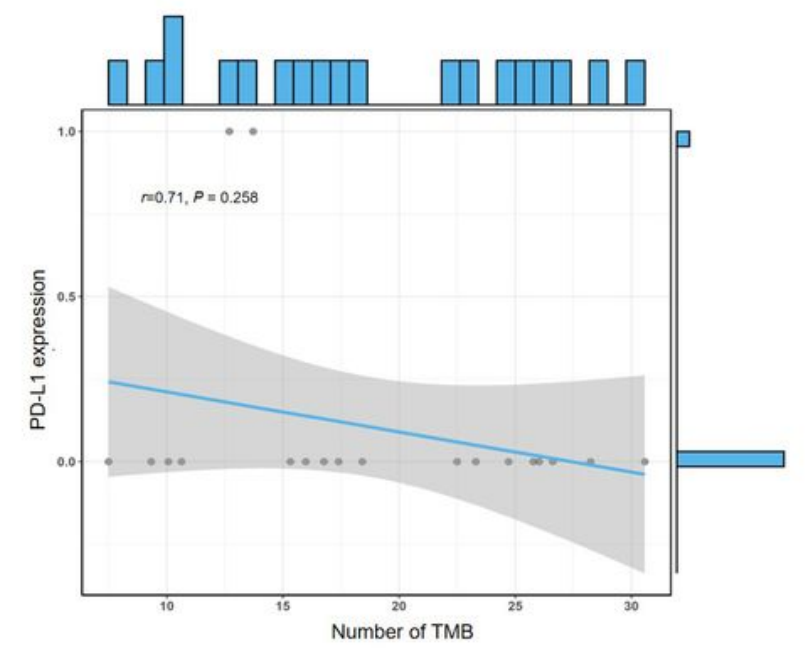


Tumour mutational burden and neoantigens in patients with LC\&O/CTB, LC\&ATB, or LC. (A) Violin plots showing the TMB in each patient from the LC\&O/CTB, LC\&ATB, and LC groups. Significant inter-group differences in TMB were detected using the Kruskal-Wallis test $(P=0.0041)$. (B) Violin plots showing the TMB in each patient from the LC\&TB and LC groups. A significant inter-group difference in TMB was detected using the two-sided Wilcoxon rank-sum test $(P=0.00062)$. (C) Violin plots showing the differences in clinical characteristics between the LC\&TB and LC groups. (D) The number of neoantigens was strongly correlated with the number of nonsynonymous mutations in the 40 patients $(r=0.74, P<$ 0.01). (E) The PD-L1 expression was not significantly correlated with TMB in the 40 patients $(r=0.71, P=$ 0.258). (F) Boxplots showing the number of neoantigens in each patient from the LC\&O/CTB, LC\&ATB, and LC groups. (G) Violin plots showing the number of neoantigens in each patient from the LC\&TB and LC groups. 
A
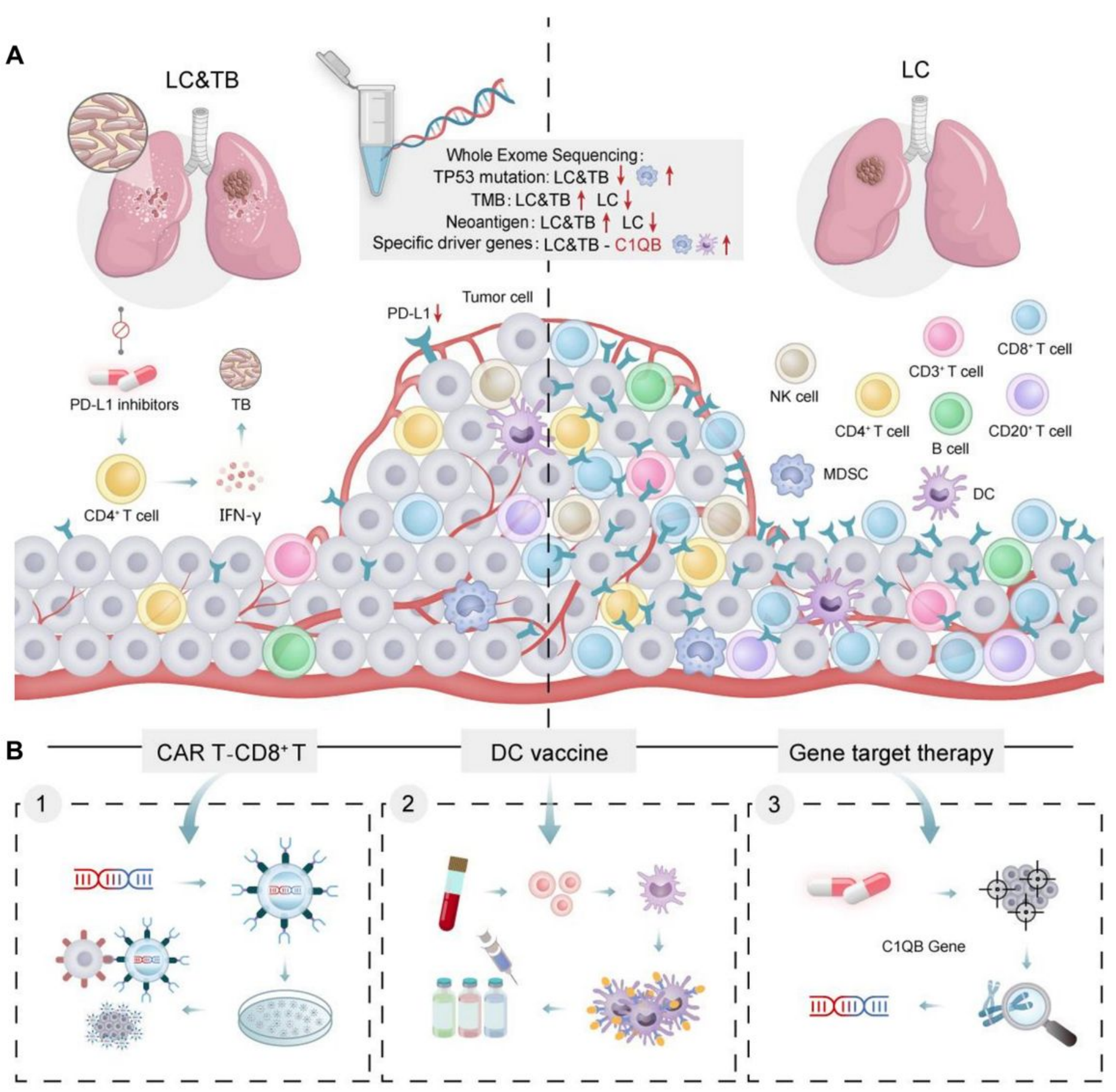

\section{Figure 6}

A summary of the study's conclusions $(A)$ and potential future treatments (B).

\section{Supplementary Files}

This is a list of supplementary files associated with this preprint. Click to download.

- SupplementaryFigureS1.jpg

- SupplementaryFigureS2.jpg 
- SupplementaryFigureS3.jpg

- SupplementaryFigureS4.jpg

- SupplementaryFigureS5.jpg

- SupplementaryFigureS6.jpg

- SupplementaryFigureS7.jpg

- SupplementaryFigureS8.jpg

- SupplementaryFigureS9.jpg

- SupplementaryFigureS10.jpg

- SupplementaryFigureS11.jpg

- SupplementaryFigureS12.jpg

- SupplementaryMaterials.docx

- SupplementaryTables.xls 